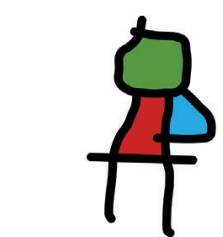

SEICAIP

\section{Allergologia et immunopathologia}

Sociedad Española de Inmunología Clínica, Alergología y Asma Pediátrica

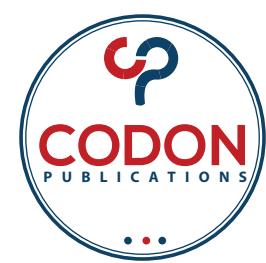

POINT OF VIEW

\title{
Oral allergy syndrome by fruit and vegetable PR-10 allergy: Accuracy of in vivo diagnosis
}

\section{Cristina De Rose ${ }^{a}$, Maria Letizia Pattia ${ }^{a}$ Alessandro Gambacorta ${ }^{a}$, Federica Brancato ${ }^{a}$, Stefano Miceli Sopo ${ }^{\text {b* }}$}

aDepartment of Life Sciences and Public Health, Policlinico Gemelli Universitary Foundation IRCCS, Catholic University of the Sacred Heart, Rome, Italy

${ }^{b}$ Allergy Unit, Pediatrics Area, Department of Life Sciences and Public Health, Policlinico Gemelli Universitary Foundation IRCCS, Catholic University of the Sacred Heart, Rome, Italy

Received 15 March 2020; Accepted 5 May 2020

Available online 1 March 2021

\section{KEYWORDS}

Component-resolved

diagnosis;

oral allergy

syndrome;

prick-by-prick;

PR-10 allergy;

skin prick test.

\begin{abstract}
Routine diagnostic methods for allergies to plant-derived foods are based on skin prick test (SPT) with commercial extracts, prick-by-prick (PbP) with fresh food, serum-specific IgE measurement, and oral food challenge.

We discuss the possibility and the advantages of performing, in patients with oral allergy syndrome (OAS) by fruit and vegetables (excluding nuts) PR-10 allergy, component-resolved diagnosis (CRD) by SPT and PbP with raw and cooked vegetables, rather than performing a CRD with in vitro tests by drawing blood.

Based on our clinical experience and the studies published in the literature, we believe that, at least for the OAS by fruit and vegetables (excluding nuts) PR-10 allergy, the search for sensitizing allergens and related cross-reactive allergens with SPT and PbP can be performed routinely in clinical practice, even at the primary-care level.
\end{abstract}

(c) 2021 Codon Publications. Published by Codon Publications.

*Corresponding author: Stefano Miceli Sopo, Department of Life Sciences and Public Health, Policlinico Gemelli Universitary Foundation IRCCS, Catholic University of Sacre Hearth, Rome 00168, Italy. Email address: stefano.micelisopo@unicatt.it; stefano.micelisopo@policlinicogemelli.it 


\section{Introduction}

Fruits and vegetables are a fundamental part of the western world's diet, but can cause food allergies. The main allergens are highly cross-reacting allergens. Among these, profilin ${ }^{1}$ and Bet $\vee 1$ proteins homologous (pathogenesis-related proteins, PR10) ${ }^{1}$ are extremely pepsin-sensitive; the nonspecific lipid-transfer protein (LTP) 1,2 and seed storage protein (SSP) ${ }^{3}$ are heat- and pepsin-stable. There are proteins with homologous structures to each other that are found not only in food but also in pollens and/or latex. This leads to a wide spectrum of lgE-mediated cross-reactions between the different members of fruit and vegetable families and between the same foods and pollen and/or latex. ${ }^{4}$ In virtually all sensitized patients, pepsin-sensitive proteins induce only mild local symptoms (oral allergy syndrome, OAS), whereas more stable allergens reach the gastrointestinal tract in a biologically active form and may induce potentially systemic symptoms.

For a clinician, this scenario is complicated by the fact that subjects sensitized to stable allergens may have both mild (OAS) and/or systemic symptoms, in particular, with regard to LTP allergy.1 Moreover, the scenario becomes further complicated by the presence of some unstable allergens (profilin and PR-10) that are found in some foods (such as celery, soy, peanuts, and roasted hazelnuts), which have different behavior compared to the usual one, with the risk of having systemic reactions. ${ }^{5,6}$ Available routine diagnostic methods for allergies to plant-derived foods, namely skin prick test (SPT) with commercial extracts, prick-byprick $(\mathrm{PbP})$ with fresh food, specific IgE measurement with extracts, and oral food challenge (OFC), are often very sensitive, but none of them gives us any information about the allergenic molecule(s) causing the sensitization. ${ }^{5}$

In subjects with OAS, it is useful to perform the search for specific IgE for both suspected food allergens, as diagnostic confirmation, and foods containing cross-reactive molecules, in order to program OFC and provide dietary advice. For example, it is important to know whether patients with OAS are sensitized to LTPs or SSDs, because in this case, they are at a risk of experiencing systemic reactions. ${ }^{4}$ In this context, component-resolved diagnosis (CRD) of allergy allows the identification of the allergens involved to determine the patient's allergenic profile by decreasing the related risks and helping to identify the most effective dietetic advice to improve patient's quality of life. ${ }^{1}$

In view of the high costs and limited accessibility to the CRD performed in the laboratory, 7,8 an aspect that is worth discussing is the advantage of performing CRD by SPT with commercial extracts and by $\mathrm{PbP}$ with fresh-cooked and raw foods (with both peel and pulp), rather than performing a molecular in vitro analysis. Some studies ${ }^{1,9}$ have demonstrated the importance of performing a CRD in vivo. Asero et al. ${ }^{1}$ evaluated the effectiveness of CRD of plant-food allergy by means of SPT with extracts of plant-derived foods containing one single allergen protein due to either the loss of labile allergens during the preparation process or a proper purification procedure of relevant protein.

\section{Case report (see also Table 1)}

Case 1. F. is a seven-year-old girl who came to our observation in the spring of 2018 with reported perioral erythema,
Table 1 History, skin prick test, prick-by-prick, and component-related diagnostic in case 1 and 2 .

\begin{tabular}{lll}
\hline History & Case 1 & Case \\
\hline Is oral allergy syndrome always regressed & Yes & Yes
\end{tabular}

after 5-10 min, sometimes after oral antihistamine administration to relieve the discomfort?

Is peeled fruit tolerated better than fruit No No with peel?

Symptoms from commercial juices or heat- No No processed food

OAS eating melon, watermelon, tomato, No No and citrus fruits

Systemic symptoms

$\mathrm{SPT}$ and $\mathrm{PbP}$

SPT with birch pollen (Lofarma)

SPT with (ALK) profilin

SPT with commercial (ALK) peach extract

SPT with hazel pollen (Lofarma)

SPT with other seasonal airborne allergens Neg Pos

(Lofarma)

SPT with commercial hazelnut extract Pos Pos

(Lofarma)

SPT with commercial walnut extract Neg Neg

(Lofarma)

SPT with commercial peanut extract Neg Neg

(Lofarma)

$\mathrm{PbP}$ with raw fresh vegetables (peel and Pos Pos

pulp separately): apple, pear, grapes, fennel, carrot, peach, apricot, cherry, strawberry, medlar, plum, and kiwi

$\mathrm{PbP}$ with raw celery and pasteurized soymilk

$\mathrm{PbP}$ with cooked vegetables (peel and pulp separately): apple, pear, grapes, fennel, carrot, peach, apricot, cherry, strawberry, medlar, plum, kiwi, and celery

$\mathrm{PbP}$ with natural rubber latex (glove) Neg Neg

In vitro CRD

Pos Pos

hazelnut, and soy

Specific IgE for profilin: birch, peach, Neg Neg hazelnut, and soy

Specific IgE for LTP: peach, hazelnut, Neg Neg and soy

Specific IgE for SSP: soy and peanut

Neg Neg

CRD: component-related diagnostic; Neg: negative; OAS: oral allergy syndrome. SPT: skin prick test; PbP: prick-byprick; PR10: plant defense proteins; Pos: positive; LTP: lipid transfer protein; SSP: seed storage protein.

labial edema, itching in the pharynx about 15 min after eating a raw peeled pear. She had eaten peeled pear without any problem many times before. Since the spring of 2017 , she has had a history of rhinitis. In September 2017, she had presented an episode characterized by perioral erythema after eating an apple that was peeled too. In May 
2018, the girl presented the same symptoms after eating grapes and one more time after eating carrot and fennel salad. In the history, it is reported that the family has a country house surrounded by hazel trees.

Case 2. A. is a 10-year-old girl with seasonal allergic rhinitis (spring-summer), sometimes associated with conjunctivitis, bronchial asthma, and atopic dermatitis. In May 2017, she presented the first clinical manifestation localized in the oropharyngeal area with a throat itch, following cherry ingestion. In May 2018, she presented the same symptoms after eating raw peeled apple, raw peeled peach, raw apricot, raw peeled pear, and raw carrots. However, before 2017, she had always eaten these foods without any symptoms. She never ate foods containing soya and celery.

For both patients, based on the clinical history, the outcome of the SPT with commercial extracts and PbP (with raw and cooked-boiled vegetables for $20 \mathrm{~min}$ ), we suspected pollen-food syndrome caused by PR-10 allergy. The patients were first allergic to pollens and then to fruit that contains an unstable allergen similar to that of the birch (Betv-1, PR-10). CRD in vitro results were consistent with this diagnostic hypothesis. Furthermore, to confirm the diagnosis and to liberalize food, we performed OFC with all the foods for which the children were found to be sensitized in order to assess the hypothetical risk of systemic reactions. In particular, we performed OFC with the foods that contained a form of PR-10 that could have different behaviors, giving rise to systemic reactions (soy and celery). After the ingestion of these foods, F. had only local reactions, for which all foods were liberalized. In addition, A. had only local reactions after the ingestion of these foods, except for raw celery. In fact, after ingestion of progressively increasing quantities, the girl also presented, besides itching in the oral cavity and throat, abdominal pain. We therefore indicated to eliminate celery from the diet. The OFC with cooked celery for A. was refused by parents. Written and oral consents from the patients and their parents were obtained, and the study was approved by the local ethical committee.

\section{Discussion}

For our patients we have highlighted a correlation between clinical history, SPT, and PbP. With these three diagnostic investigations and, at a second level, with OFC, we have produced an accurate diagnosis for both patients, performing an adequate risk assessment essential for the clinical management of allergic patients. CRD performed by blood sampling only confirmed what was detected by SPT and PbP.

With regard to the opportunity to perform OFC, we believe that it is clinically relevant for these patients in order to avoid a possibly improper elimination diet. Several reasons may explain the importance of OFC within the diagnostic and therapeutic pathways of OAS if CRD is performed both in vivo and in vitro: (1) having a positive SPT for a food or the presence of specific IgE means that the patient is sensitized, but this does not imply clinical allergy, for example, in the case of sensitization to LTP, foods containing LTP should be avoided only if important symptoms of exposure appear; (2) the level of serum-specific IgE and the size of the SPT and/or PbP wheal cannot predict the dose of the food that induces the clinical reaction and the severity of the same reaction; (3) the exceptions to the usual behavior of labile allergens must always be taken into consideration (as a particular form of PR-10 that can present in soy and raw celery and can have different behaviors giving rise to systemic reactions)..$^{5,10}$ In particular, the importance of the OFC is highlighted by our Case 2, which has the particularity of having a raw celery PR-10 syndrome (one of the so-called risky foods) characterized by systemic symptoms such as abdominal pain.

In conclusion, in patients with OAS, can CRD be performed in vivo without taking blood samples? In our opinion the answer is yes, at least limitedly to OAS by fruit and vegetable (excluding nuts) PR-10 allergy (and probably also in the case of profilin allergy). We believe that the search for sensitizing allergens and related cross-reactive allergens based on SPT with extracts and PbP with raw and cooked vegetables can be performed routinely in clinical practice even at the primary-care level, with important advantages in terms of both clinical care (immediate results) and cost reduction.

\section{Ethical statement}

We declare that the work described has not been published previously, it is not under consideration for publication elsewhere, its publication is approved by all authors and tacitly by the responsible authorities where the work was carried out, and, if accepted, it will not be published elsewhere in the same form, in English or in any other language, including electronically, without the written consent of the copyright holder.

\section{Conflict of interest}

None

\section{Funding}

None. This research did not receive any specific grant from funding agencies in the public, commercial, or not-forprofit sectors.

\section{Author contributions}

SMS conceived the design of the study. CDR drafted the article. MLP, AG, and FB acquired the data and researched the scientific literature. CDR and SMS analyzed and commented on it. All authors revised the article and gave final approval to the version to be published.

\section{References}

1 Asero R, Jimeno L, Barber D. Component-resolved diagnosis of plant food allergy by SPT. Eur Ann Allergy Clin Immunol. 2008;40(N4):115-121.

2 Asero R, Mistrello G, Roncarolo D, de Vries SC, Gautier MF, Ciurana $\mathrm{CL}$, et al. Lipid transfer protein: A pan- allergen in plant-derived foods that is highly resistant to pepsin 
digestion. Int Arch Allergy Immunol. 2000;122:20-32. https:// doi.org/10.1159/000024355

3 Lehmann K, Schweimer K, Reese G, Randow S, Suhr M, Becker $W M$, et al. Structure and stability of $2 S$ albumin-type peanut allergens: Implications to the severity of peanut allergic reactions. Biochem J. 2006;395:463-72. https://doi.org/10.1042/ BJ20051728

4 Ebisawa M, Ballmer-Weber BK, Vieths S, Wood RA (eds). Food allergy: Molecular basis and clinical practice. Chem Immunol Allergy.2015;101:162-70. https://doi.org/10.1159/isbn.978-3-31802341-1

5 Asero R. Plant food allergies: A suggested approach to allergen-resolved diagnosis in the clinical practice by identifying easily available sensitization markers. Int Arch Allergy Immunol. 2005;138:1-11. https://doi.org/10.1159/000087352

6 Asero R, Celi G, Scala E. Labile plant food allergens: Really so harmless? Case series and literature review. Allergy. 2020 Jun;75(6):1517-1518. https://doi.org/10.1111/all.14184
7 Alessandri C, Ferrara R, Bernardi ML, Zennaro D, Tuppo L, Giangrieco I. Diagnosing allergic sensitizations in the third millennium: Why clinicians should know allergen molecule structures. Clin Transl Allergy. 2017;17(7):21. https://doi. org/10.1186/s13601-017-0158-7

8 Tuppo L, Giangrieco I, Alessandri C, Ricciardi T, Rafaiani C, Ciancamerla $M$, et al. Pomegranate chitinase III: Identification of a new allergen and analysis of sensitization patterns to chitinases. Mol Immunol. 2018;103:89-95. https://doi.org/ 10.1016/j.molimm.2018.09.009

9 Asero R, Monsalvew R, Barber D. Profilin sensitization detected in the office by skin prick test: A study of prevalence and clinical relevance of profilin as a plant food allergen. Clin ExperimAllergy. 2008;38:1033-7. https://doi.org/10.1111/j.13652222.2008.02980.x

10 Webber CM, Enland RW. Oral allergy syndrome: A clinical, diagnostic and therapeutic challenge. Ann Allergy Asthma Immunol. 2010;104:101-8. https://doi.org/10.1016/j.anai.2009.11.007 